\title{
Apresentação
}

\section{Pagamento por desempenho, formulação, implementação e sucesso da política em países de renda baixa, média e alta ${ }^{1}$}

\author{
Fabiana da Cunha Saddi ${ }^{2}$ \\ Professora Doutora Visitante da Universidade \\ Federal de Goiás, Goiânia, Goiás, Brasil. \\ fabianasaddiı@gmail.com \\ Stephen Peckham \\ Professor de Política de Saúde da Universidade \\ de Kent, Cantuária, Inglaterra \\ s.peckham@kent.ac.uk \\ Pedro dos Santos Mundim \\ Professor Doutor da Universidade Federal de Goiás, \\ Goiânia, Goiás, Brasil \\ psmundim@gmail.com
}

Países de renda alta (PRA), baixa e média (PRBM) inseridos em distintos contextos político-econômicos e de dependência (ou não) do financiamento externo, bem como em estágios diferenciados de desenvolvimento da política de atenção primária à saúde (APS), têm adotado programas de pagamento por desempenho ou de financiamento baseado em desempenho (P4P/FBD) na APS. Levando em conta a realidade de sistemas e contextos de saúde nos países, os pressupostos racionais que caracterizam o desenho de programas de $\mathrm{P} 4 \mathrm{P} / \mathrm{FBD}$ têm sido teoricamente questionados por pressupostos políticos, organizacionais e motivacionais mais realistas. Empiricamente, e de forma distinta entre os países, eles têm

1 Agradecemos a Germano Coelho e Jaqueline Damasceno pela ajuda na edição desta apresentação.

2 Fabiana da Cunha Saddi também é pesquisadora visitante na Universidade de Kent e, juntamente com Stephen Peckham, é Newton Advanced Fellow da Academia Britânica e Fundo Newton. 
sido desafiados por lógicas políticas alternativas decorrentes de processos de implementação e reformulação comparativamente distintos, de diversos efeitos (ou evidências) inesperados(as) e do reconhecimento da necessidade de se efetuar adaptações e considerar impactos de longo prazo (sustentáveis) nos sistemas de saúde.

Este dossiê, intitulado "Pagamento por desempenho, formulação, implementação e sucesso da política" reúne artigos de PRBM e a tradução de um artigo sobre o Quality and Outcomes Framework (QOF) adotado na Inglaterra/ Reino Unido. O intuito é reunir trabalhos brasileiros e internacionais, e em especial, devido à lacuna existente na literatura brasileira, sobre o Programa Nacional de Melhoria do Acesso e da Qualidade da Atenção Básica (PMAQ), que constitui, em sua maioria, análises quantitativas dos dados do programa (Saddi; Peckham, 2018). Dada a grande variedade de programas de P4P/PBF em PRBM e PRA, desenhados e implementados de forma separada nos países, este dossiê apresenta-se como oportunidade para efetuar uma avaliação comparativa desses programas.

Programas de $\mathrm{P} 4 \mathrm{P} / \mathrm{FBD}$ podem ser amplamente definidos como esquemas que introduzem incentivos financeiros para trabalhadores/provedores ou formas de financiamento parcial de sistemas de saúde com base em critérios de desempenho. $\mathrm{Na}$ maioria dos países de renda baixa (PRB), o P4P é conhecido como financiamento baseado em desempenho (FBD). Este requer a realização de contratos com unidades de saúde sob a responsabilidade dos governos. $\mathrm{O}$ FBD, em sua fase inicial, ainda não consiste em um programa do sistema de saúde do país. O FBD foi desenhado por um grupo de experts, teve sua origem em um projeto empreendedor no Camboja, passando a receber o apoio e a ser executado pelo Banco Mundial e outras agências de fomento quando iniciava sua adoção na África (Fritsche; Soeters; Meessen, 2014).

No Brasil, o PMAQ consiste em um programa nacional elaborado por formuladores do Departamento de Atenção Básica do Ministério da Saúde. Surgiu num contexto em que havia a necessidade de aprimorar a avaliação da Estratégia Saúde da Família, a fim de justificar e garantir o aumento do financiamento na APS (Saddi; Pego, 2018). No Reino Unido, o QOF fez parte de um novo contrato nacional com clínicos gerais (CG), introduzido em 2004, como uma resposta a anos de falta de investimento na clínica geral em comparação com outras partes do serviço de saúde, baixa moral entre CG e variações na qualidade do atendimento médico. Embora fosse voluntário participar, a grande maioria dos CG aproveitou a oportunidade para obter renda adicional. De forma semelhante entre os PRBM e PRA, a maioria dos esquemas objetivam melhorar os cuidados na APS, desenvolver padrões nacionais, regionais e locais comparáveis de qualidade. Nos PRBM, os P4P/FBD também visam melhorar o acesso aos serviços. Nos PRB, o FBD também é utilizado na reconstrução de sistemas de saúde ou melhoria de indicadores epidemiológicos ou básicos de saúde.

Brasil e países africanos possuem, ou estão desenvolvendo, uma APS de base comunitária, atuando com equipes de saúde compostas por médico, enfermeiras e agentes comunitários de saúde (ACS). Nos países africanos, há níveis distintos de integração comunitária e de ACS nos serviços - embora o envolvimento do ACS seja considerado 
um fator importante na implementação do FBD, como aconteceu em Ruanda. No Brasil, o PMAQ/P4P é adotado num tempo avançado do desenvolvimento APS, tendo em vista a extensão da ESF no território nacional. Entretanto, o PMAQ não conseguiu incluir o trabalho do ACS durante sua implementação (Saddi et al., 2017; Saddi; Pego, 2018). Na Inglaterra, o QOF está ligado ao trabalho do clínico geral e seus serviços contratados.

No que diz respeito ao processo de formulação e desenho, o P4P/FBD ainda é pouco estudado em todo o mundo. Nos PRB isto possivelmente se deve ao fato da formulação não ocorrer inicialmente no país ou não ser liderada pelo governo. Estudo realizado em 12 países (Shroff; Bigdeli; Meessen, 2017) apresenta algumas facilidades e barreiras para os países avançarem para as fases de adoção nacional e institucionalização do FBD/P4P. Revisões e análises (Eijkenaar, 2013; Paul et al., 2018; Renmans et al., 2016), embora distintamente, chamam a atenção para a necessidade de se levar em conta o contexto e desenho, bem como ressaltam que é preciso compreender melhor como atores nacionais promovem o FBD (se tornam campeões do FBD) e influenciam a formulação (Gautier; Ridde, 2017). Todos eles enfatizam a necessidade de se levar em contar os atores/instituições nacionais.

No Brasil sabe-se que o PMAQ foi elaborado pela equipe do Departamento de Atenção Básica do Ministério da Saúde, discutido com membros de conselhos nacionais de saúde no grupo de trabalho, em que se verificou a formação de consenso sobre o programa, que seguiu para aprovação na Comissão Intergestores Tripartite (Saddi; Pego, 2018). Não sabemos ainda de forma mais analítica e em profundidade como se deu o processo de formulação do PMAQ nos distintos ciclos e como atores nacionais pesquisadores, conselheiros e outros-e experts internacionais têm influenciado o re-desenho do programa. $\mathrm{Na}$ Inglaterra, várias análises e comentários de pesquisadores acadêmicos, consultorias, grupos de pressão de profissionais de saúde e comitês examinaram o impacto e o processo da QOF. Estudos recentes (Forbes; Marchand; Peckham, 2016; Guthrie;Tang, 2016), e uma análise do NHS (National Health System England, 2018), questionam o valor e a futura continuação do programa. Mesmo assim, ainda não localizamos um estudo focado na formulação do QOF desde sua implementação em 2004.

Em relação à implementação, temas como capacidade organizacional, engajamento do pessoal, motivações profissionais, estresse e sobrecarga de trabalho também são amplamente considerados tanto em PRA (Guthrie; Tang, 2016; Peckham, 2007) quanto em PRBM (Eijkenaar, 2013; Renmans et al., 2016; Saddi et al., 2018; Saddi; Peckham, 2018; Toonen et al., 2009). Embora de formas variadas entre PRBM e PRA, pesquisadores levaram em consideração consequências não intencionais, como jogos e trapaça no Brasil (Saddi et al., 2017), efeitos perversos nos países africanos (Paul et al., 2018) e burocratização, autonomia clínica e profissionalismo, crowding out e jogos no Reino Unido (Guthrie; Tang, 2016).

Por se concentrar em aspectos de atendimento clínico que podem ser facilmente mensuráveis e rotineiramente coletados, pesquisadores têm questionado a forma como o impacto do programa e seu sucesso tem sido medido e prevalecente na literatura. Embora de modos distintos, pesquisadores de PRA (Peckham; Wallace, 2010) e de PRBM questionam 
até que ponto o beneficio financeiro impactou os trabalhadores e organizações locais de saúde. Os pesquisadores também questionam se esse beneficio afetou a qualidade do atendimento em áreas não consideradas pelo programa, se ele foi eficaz para melhorar o desempenho ou se pode ser considerado essencial para a manutenção da qualidade do cuidado. Evidências em termos de eficácia e eficiência do FBD são variadas entre os países, e pesquisadores enfatizaram o fato de que não há evidências de que o FBD seja uma estratégia pró-pobre e, assim, precisaria de metas de equidade (Skiles et al., 2013). Tratase de consenso emergente em PRB (Ridde et al., 2018) e de debate incipiente no Brasil. Em PRB, análises/debates também ocorrem em torno da necessidade de se repensar o esquema (Paul et al., 2018), ou como estratégia/ferramenta catalizadora de uma reforma compreensiva (Meessen; Soucat; Sekabaraga, 2011; Fritsche; Soeters; Meessen, 2014) ou que pode ser institucionalizada nos sistemas e conforme barreiras/facilidades encontradas (Shroff; Bigdeli; Meessen, 2017). Evidências dos primeiros anos do QOF mostraram que o programa reduziu as variações nas práticas clínicas incentivadas e contribuiu para melhorar o uso dos registros eletrônicos e da atenção multidisciplinar liderada por enfermeiros em condições de longo prazo. No entanto, após o primeiro ano do QOF, a maioria das práticas alcançou uma remuneração quase máxima do esquema, levantando questões sobre o seu valor e, a cada ano, ajustes foram feitos nas medidas, estando agora muitos "aposentados". Mais recentemente, o esquema foi abandonado na Escócia e há uma variação crescente em sua aplicação na Inglaterra, com a possibilidade de seu uso ser abandonado em todo o país, embora os dados sobre as áreas de cuidado que estavam no QOF continuem sendo monitorados, mas não necessariamente incentivados financeiramente.

Os trabalhos incluídos nesta edição podem ser vistos como casos relevantes do estado atual do conhecimento dos diferentes contextos político-econômicos. $\mathrm{O}$ artigo de discussão de Pierre Abomo chama a atenção para o fato da literatura de FBD ainda não ter explorado suficientemente as implicações relativas às mudanças ideológicas e operacionais que o FBD está ocasionando. Introduz o conceito de despolitização no debate sobre FBD e, em especial, relacionado ao caso de Camarões, para descrever a capacidade do FBD para redesenvolver uma política de saúde que leve em conta a intervenção política e estatal e a primazia do setor público em relação ao campo de competição de mercado em que se encontram o governo patrocinado e atores não estatais. $O$ autor recomenda que o FBD seja repensado em relação à forma como se dá o desenho, a introdução e a implementação, incorporando atores nacionais no desenho da ferramenta e definição dos objetivos e indicadores. Enfatiza que o FBD, como proposto por atores externos, precisa ser imerso nas práticas e processo de formulação do país.

Lara Gautier e Valéry Ridde efetuam uma análise documental da agenda de aprendizagem do Fundo Fiduciário de Inovação em Resultados para a Saúde (HRITF) do Banco Mundial ao longo de dez anos, em que se verificou a predominância de avaliações de impacto de programas pilotos de FBD adotados em PRBM. Os autores mostram que as produções/ações do HRITF produziram tanto uma forma de conhecimento politizado como um uso transformador do conhecimento na área, tendo em vista a forma 
como projetos pilotos financiados pelo Fundo foram desenhados e avaliados. Atividades de aprendizado diversas ocasionaram um uso transformador do conhecimento para os funcionários do Banco Mundial, implementadores e formuladores de políticas nacionais. Os autores recomendam que a instituição precisa avançar para se tornar uma organização de aprendizagem transformadora.

No artigo "Programa Nacional de Melhoria do Acesso e da Qualidade da Atenção Básica (PMAQ-AB): modelização da política no âmbito nacional”, Larissa Lemos, Nília Maria Prado e Maria Guadalupe Medina realizam uma sistematização de elementos conceituais do desenho do PMAQ no Brasil. Com esta sistematização, as autoras não apenas assinalam a plausibilidade lógica da proposta, como esperam que a mesma possibilite uma ampliação da avaliação para aspectos mais organizacionais. A modelização viabilizará uma comparação entre o desenho e a implementação do programa. Trata-se de trabalho relevante no contexto atual de avaliações do PMAQ, por trazer elementos que vêm à tona na implementação e redesenho do programa.

Jessica Gergen, Yogesh Rajkotia e Nirmala Ravishankar trazem uma contribuição debatendo se o FBD mostra-se como mecanismo capaz de produzir transformações amplas nos sistemas de saúde. Os autores utilizam-se de métodos qualitativos para analisar a implementação do FBD em unidades de saúde, verificando a forma como se deu a promoção de efeitos positivos, perversos e disruptivos sobre o sistema de saúde em duas províncias de Moçambique. Dentre os resultados positivos encontram-se o aumento da autonomia, da capacidade fiscal local e do planejamento. Dentre os negativos, observou-se que a alocação de incentivos entre os funcionários foi injusta e sem transparência, houve incremento da carga de trabalho e tempo despendido com dados no sistema de informações, em detrimento do atendimento clínico. $\mathrm{O}$ estudo demonstra que resultados positivos na institucionalização de mudanças na governança, nas estruturas de gestão e na autonomia fiscal, só ocorrem na medida em que se verifica um aumento na inclusão do lado da demanda.

Thabata Zermiani, Marcia Pinto e Rafael Ditterich efetuam um estudo de tipo qualitativo e quantitativo para analisar a percepção dos trabalhadores da saúde de CuritibaPR entre 2002-2015, no Brasil, em relação ao uso de incentivos profissionais como ferramenta na gestão e organização da APS. O estudo foca no programa Incentivo ao Desenvolvimento da Qualidade (IDQ) adotado pela Secretaria Municipal de Saúde. Os autores demonstram que o incentivo inicialmente teve resultados positivos, impactando na motivação dos profissionais, na qualidade do trabalho e no alcance das metas. Contudo, mostram que com o tempo a avaliação se tornou uma rotina, não sendo realizada da forma preconizada. O processo avaliativo, a priorização da quantidade, a fraude de informações no atingimento de metas e o fato dos incentivos serem empregados para não haver aumento salarial foram considerados elementos que dificultaram a implementação do programa. $\mathrm{Na}$ autoavaliação dificilmente os profissionais atribuíam nota inferior a dez. Autores destacam que o P4P deve ser transparente e imparcial, promover o diálogo entre profissionais e possibilitar que gestores/profissionais participem da pactuação para que 
haja impacto efetivo na qualidade/resultados. Este estudo traz lições que podem e têm sido consideradas em poucas análises de implementação do PMAQ.

Alline Orué, Albert Souza, Alcindo Ferla, Débora Nascimento e Mara Santos utilizam dados secundários do segundo ciclo do PMAQ para analisar a implantação, o planejamento e o monitoramento do Núcleo de Apoio à Saúde da Família (NASF). Os dados do PMAQ permitiram verificar que o NASF foi implantado sem a devida articulação com a APS, sendo centralizado na gestão, principalmente nas capitais. $\mathrm{O}$ planejamento foi organizado, periódico e articulado, e o monitoramento ainda é uma atividade pouco desenvolvida no Brasil, com exceção do caso do PMAQ, que induz atividades mediante incentivo financeiro proporcional à nota das equipes/participantes do programa e possibilita o monitoramento. Este estudo exemplifica a forma como os dados do PMAQ têm sido utilizados em todo país para analisar ou monitorar processos, efeitos relacionados à APS brasileira, resultando em diversos trabalhos. Não são, contudo, utilizados necessariamente para analisar a implementação e os processos do PMAQ ou aspectos concernentes estritamente ao programa ou a temas de P4P.

Martin Roland e Frede Olesen, no artigo "O pagamento por desempenho pode melhorar a qualidade da atenção primária de saúde?”, originalmente publicado no BMJ e traduzido em português neste dossiê, exploram o que outros países podem aprender com a experiência do Quality and Outcomes Framework (QOF) do Reino Unido. Os autores trazem lições sobre como a qualidade da atenção tem sido e deve ser medida, e sobre quem deve decidir sobre quais indicadores adotar. Problematizam ainda o fato de que médicos não deveriam excluir pacientes por causa de alvos do programa e, assim, indagam se o desempenho é a forma mais útil de se pagar os médicos. Refletem também sobre com que frequência um esquema de pagamento de desempenho deve ser revisado e assinalam o papel das consequências não intencionais para a prática e formuladores/ gestores. Tratase de temas que têm caracterizado a adoção do QOF, mas que se mostram comuns a $\mathrm{P} 4 \mathrm{P} / \mathrm{FBD}$ em outras partes do globo. Trazem luzes sobre o processo de formulação e implementação em países que adotam modelos de base comunitária na APS.

A entrevista com James Macinko, concedida a Fabiana C. Saddi, sistematiza o conhecimento e a opinião de estudioso renomado da área sobre o PMAQ, lançando luzes sobre processos e temas/subtemas politicamente relevantes neste estágio de implementação do PMAQ no Brasil. Macinko fala sobre a origem e a relevância do PMAQ na APS brasileira, bem como de iniciativas de incentivo que antecederam o PMAQ. Macinko ainda responde questões sobre riscos que podem surgir da introdução do $\mathrm{P} 4 \mathrm{P}$ no sistema de saúde, sobre resultados produzidos até o momento, lições aprendidas e recomendações para avançar com o PMAQ. Por fim, o entrevistado ressalta como as Ciências Sociais brasileira poderiam contribuir para aumentar nosso conhecimento sobre o PMAQ. Dentre os principais subtemas ressaltados destacam-se: o fato de municípios com melhores recursos terem mais condições de ganhar o incentivo; o incremento de dados sobre a APS e usos para vários fins; necessidade de tornar o atual método de coleta de dados mais transparente e independente; necessidade de maior 
compreensão da maneira como os incentivos estão sendo usados e de comunicar o trabalho da ESF aos usuários e formuladores.

\section{Referências}

EIJKENAAR, Frank. Effects of pay-for-performance in health care: a systematic review of systematic reviews. Pay-for-Performance for Healthcare Providers Design, performance measurement, and (unintended) effects. Netherlands: Het instituut Beleid \& Management Gezondheidszorg, 2013. Available from: <https://www.bmg.eur.nl/fileadmin/ASSETS/bmg/Onderzoek/Promoties/Promoties_2013/Frank_ Proefschrift.pdf>. Access on: 28 febr. 2018.

FORBES, Lindsay; MARCHAND, Catherine; PECKHAM, Stephen. Review of the Quality and Outcomes Framework in England: Final Report 2016. London: POLICY RESEARCH UNIT IN COMMISSIONING AND THE HEALTHCARE SYSTEM (PRUComm), 2016. 49 p. (Report). Available from: <http://blogs.lshtm.ac.uk/prucomm/files/2017/02/Review-of-QOF-21stDecember-2016.pdf>. Access on: 15 jan. 2017.

FRITSCHE, György Bèla;SOETERS, Robert; MEESSEN, Bruno. Performance-based financing toolkit. The World Bank, 2014. Available from: <https://openknowledge.worldbank.org/handle/10986/17194>. Access on: 12 febr. 2018.

GAUTIER, Lara; RIDDE, Valéry. Health financing policies in Sub-Saharan Africa: government ownership or donors' influence? A scoping review of policymaking processes. Global health research and policy, v. 2, n. 1, p. 23-39, 2017. Available from: <https://ghrp.biomedcentral.com/articles/10.1186/ s41256-017-0043-x>. Access on: 04 jan. 2018.

GUTHRIE, Bruce;TANG, Jason. What did we learn from 12 years of the Quality and Outcomes Framework?. Scottish School of Primary Care - Series Literature Review. September/2016. Available from: <http:// www.sspc.ac.uk/media/media_486342_en.pdf>.Access on: 23 may. 2018.

MEESSEN, Bruno; SOUCAT, Agnès; SEKABARAGA, Claude. Performance-based financing: just a donor fad or a catalyst towards comprehensive health-care reform?. Bull World Health Organ, v. 89, n. 2, p. 153-156, 2011. Available from: <https://www.ncbi.nlm.nih.gov/pubmed/21346927>. Access on: 04 mar. 2016.

NATIONAL HEALTH SYSTEM ENGLAND. Report of the Review of the Quality and Outcomes Framework in England. London: NHS England, 2018. 76 p. (Report). Available from: <https://www.england.nhs. $\mathrm{uk} /$ publication/report-of-the-review-of-the-quality-and-outcomes-framework-in-england/ $>$. Access on: 20 sep. 2018.

PAUL, Elisabeth et al. Performance-based financing in low-income and middle-income countries: isn't it time for a rethink?. BMJ Global Health, v. 3, p. 1-7, 2018. Available from: <https://gh.bmj.com/ content/3/1/e000664>. Access on: 12 aug. 2018.

PECKHAM, Stephen. The new general practice contract and reform of primary care in the United Kingdom. Healthcare Policy, v. 2, n. 4, p. 34-48, 2007. Available from: <http://researchonline.lshtm. ac.uk/4933/1/Policy_vol2_no4_peckham.pdf>.Access on: 04 may. 2018.

PECKHAM, Stephen; WALLACE, Andrew. Pay for performance schemes in primary care: what have we learnt?. Quality in Primary Care, v. 18, n. 2, p. 111-116, 2010. Available from: <http://primarycare. imedpub.com/pay-for-performance-schemes-in-primary-care-what-have-we-learnt.php?aid=904>. Access on: 08 dec. 2015.

RENMANS, Dimitri et al. Opening the "black box" of performance-based financing in low-and lower middle-income countries: a review of the literature. Health Policy Planning, v. 31, n. 9, p. 1297-1309, 2016. Available from: <https://www.ncbi.nlm.nih.gov/pubmed/27126200>. Access on: 09 aug. 2018. 
RIDDE, Valéry et al. Performance-based Financing in Africa: Time to Test Measures for Equity. International Journal of Health Services, v. 48, n. 3, p. 549-561, 2018. Available from: <https://www.ncbi. nlm.nih.gov/pubmed/29932352>. Access on: 22 sep. 2018.

SADDI, Fabiana da Cunha et al. Perceptions and evaluations of front-line health workers regarding the Brazilian National Program for Improving Access and Quality to Primary Care (PMAQ): a mixedmethod approach. Cad. Saúde Pública, Rio de Janeiro, v. 34, n. 10, e00202417, 2018. Available from: $<$ http://www.scielo.br/scielo.php?script=sci_arttext\&pid=S0102-311X2018001005009\&lng=en\&nr $\mathrm{m}=$ iso $>$. Access on: 14 nov. 2018.

SADDI, Fabiana da Cunha et al. The politics of implementing a performance measurement program (PMAQ) at the front line of primary health care in Goiania, Brazil: a qualitative political analysis. In: International Conference on Public Policy, 3., 2017. Singapore. Anais... Singapore, 2017. p. 1-35. Available from: <http://www.ippapublicpolicy.org//file/paper/59a55b90e37cd.pdf>. Access on: 02 jan. 2018.

SADDI, Fabiana da Cunha; PECKHAM, Stephen. Brazilian Payment for Performance (PMAQ) Seen From a Global Health and Public Policy Perspective. Journal of Ambulatory Care Management, v. 41, n. 1, p. 25-33, 2018. Available from: <https://www.ncbi.nlm.nih.gov/pubmed/28990991>. Access on: 10 febr. 2018

SADDI, Fabiana da Cunha; PEGO, Raquel Abrantes. The collective health movement and health policy in Brazil: from regime transition/democratization to democracy and neoliberalism (1970s to 2014). Contextualizaciones Latinoamericanas, v. 10, n. 18, p. 2-11, 2018. Available from: <http://www. revistascientificas.udg.mx/index.php/CL/article/view/7254/6390>. Access on: 18 sep. 2018.

SHROFF, Zubin Cyrus; BIGDELI, Maryam; MEESSEN, Bruno. From Scheme to System (Part 2): Findings from Ten Countries on the Policy Evolution of Results-Based Financing in Health Systems. Health Systems \& Reform, v. 3, n. 2, p. 137-147, 2017. Available from: <https://www.tandfonline.com/ doi/abs/10.1080/23288604.2017.1304190>. Access on: 20 dec. 2017.

SKILES, Martha Priedeman et al. An equity analysis of performance-based financing in Rwanda: are services reaching the poorest women?. Health Policy and Planning, v. 28, n. 8, p. 825-837, 2013. Available from: <https://academic.oup.com/heapol/article/28/8/825/581934>. Access on: 12 apr. 2017.

TOONEN, Jurien et al. Learning lessons on implementing performance based financing, from a multi-country evaluation. KIT (Royal Tropical Institute) in collaboration with Cordaid and WHO, 2009. Available from: <http://www.who.int/contracting/PBF.pdf>. Access on: 07 apr. 2016. 